\title{
CBREN: Convolutional Neural Networks for Constant Bit Rate Video Quality Enhancement
}

\author{
Hengrun Zhao, Bolun Zheng*, Shanxin Yuan, Hua Zhang*, Chenggang Yan, Liang Li, \\ Gregory Slabaugh, Senior Member, IEEE
}

\begin{abstract}
Constant bit rate (CBR) videos are widely used in streaming playback applications. However, the image quality of the CBR video is often unstable, especially for scenes with large motion. To this end, we design a new model to represent the distortion of High Efficiency Video Coding (HEVC) constant bit rate video, and propose a neural network for a constant bit rate video quality enhancement (CBREN). We propose a dual-domain restoration module (DRM) to jointly learn the prior knowledge in the pixel domain and the frequency domain. To address the degradation resulting from compression, we propose a twostep quantization degradation estimation strategy. The Inverse DCT (IDCT) Translation Unit (ITU) is used to constrain the quantization table of the constant bit rate video to a suitable range, and the Dynamic Alpha Unit (DAU) is used to finetune the quantization table according to the content of each frame. In order to effectively reduce the block distortion of different sizes produced in the compression process, we adopt a multi-scale network. Extensive experiments show that our approach can greatly enhance the quality of CBR compressed video. Moreover, our method can also be applied to constant quantization parameter (CQP) video enhancement tasks, and is certainly superior to existing methods.
\end{abstract}

Index Terms-Quality enhancement, CBR compressed video, Dual-domain restoration.

\section{INTRODUCTION}

W ITH the development of infrastructure such as largebandwidth Internet and high-performance mobile devices, digital videos are ubiquitous and the related applications are gaining momentum $[39,74]$. Because the original uncompressed video data is too large, video compression plays a key role in video transmission and storage $[45,30]$.

In order to cope with the rapid proliferation of video and improve the compression for transmission [4], a new generation of efficient video coding and compression standard, High Efficiency Video Coding (HEVC, also known as H.265) was released in 2013 [48, 46]. Compared with the existing standards, HEVC provides higher quality video images with the same bandwidth and achieves higher compression ratio with the same image quality $[64,50]$. HEVC exhibits efficient compression performance, making the transmission of video data in the network more economical and flexible [3,59], and has been well accepted by the industry since its launch [40].

H. Zhao, B. Zheng, H. Zhang, C. Yan are currently with Hangzhou Dianzi University.

* Bolun Zheng (e-mail: blzheng@hdu.edu.cn) and Hua Zhang (zhangh@hdu.edu.cn) are both the corresponding authors of this paper

S. Yuan is currently with Huawei Noah's Ark Lab.

$\mathrm{L} . \mathrm{Li}$ is currently with Institute of Computing Technology, Chinese Academy of Sciences.

G. Slabaugh is currently with Queen Mary University of London.
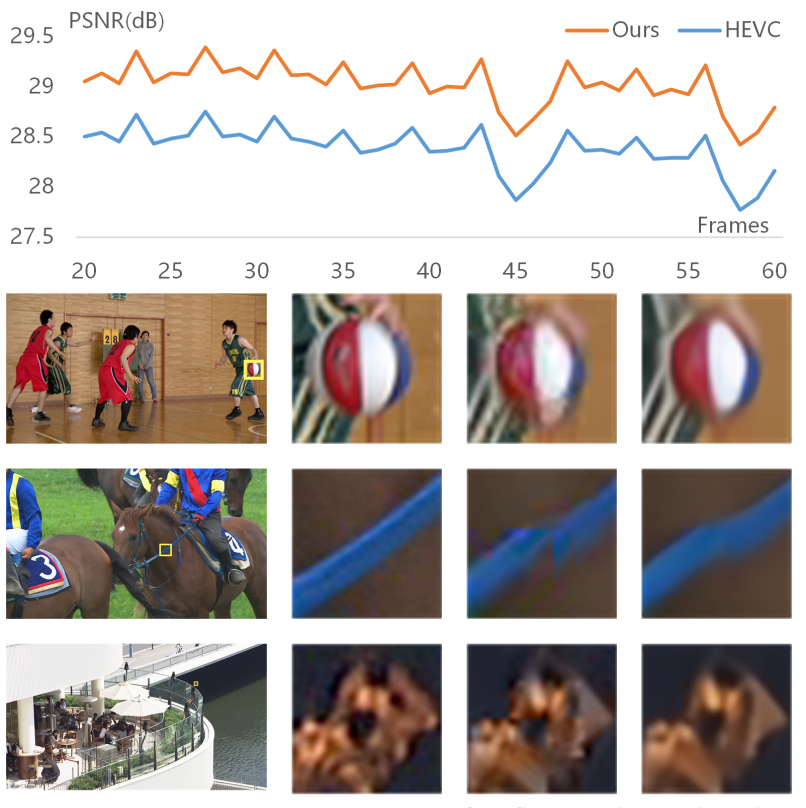

CBR Compressed Enhanced

Fig. 1: An example for quality fluctuation (top) and quality enhancement performance (bottom). The artifacts and quality of the frames in a CBR compressed video are quite unpredictable and unstable due to the motion. Our CBREN can consistently enhances visual quality of CBR video through a sequence shown by higher PSNR

Bit rate control is the process of allocating reserved bits to all levels of coding units, which underpin video coding. HEVC recommends two bit rate control strategies: constant bit rate (CBR) mode and variable bit rate (VBR) mode. The CBR mode is suitable for a scene that requires high image quality and has no strict bandwidth limit. The CBR mode, which is widely adopted in streaming playback, allows the bit rate to stay unchanged and close to the target bit rate [41]. However, to achieve the same visual quality for all images, the required bit rate varies for each image, depending on the content. Therefore using CBR mode to encode these contents with the same bit rate will lead to unstable quality, as demonstrated in Fig. 1. Constant quantization parameter (CQP) is one of the mainstream VBR control schemes. In CQP mode, all frames use the same quantization parameters, so the compression degree is roughly the same.

The video compressed by existing methods suffers from visual quality degradation, e.g., blurry boundaries, ringing 
artifacts, image distortion, and so on [56]. Traditional filterbased methods $[17,10]$ focus on general image restoration tasks, such as image denoising, super-resolution, deblocking, etc. Recently several machine-learning-based methods [7, 9, $13,47]$ have been proposed, showing better performance over traditional methods in compression artifact removal. Zhang et al. [68] propose a residual highway convolutional neural network for the in-loop filtering in HEVC. Ren et al. [60] propose the multi-frame quality enhancement (MFQE) network for video quality enhancement. Most of these approaches focus on restoring the visual quality degradation in CQP mode. Compared with CQP mode, in the CBR mode the quantization parameters $(\mathrm{QPs})$ in different coding units (CUs) are different from each other, which makes the quality of coding units fluctuate within a frame and limits the performance of CQP-oriented methods on CBR compressed video quality enhancement. Dual-domain learning attracts much attention to DCT-domain quantization based JPEG compression artifact reduction. Because the JPEG adopts a fixed $8 \times 8$ size block and quantzation table, several methods[71, 20, 67] are proposed to model the image degradation way of JPEG compression. However, unlike JPEG, the CU size and quantization table in the CBR video are all uncertain, the restoration model proposed in IDCN cannot achieve a satisfactory result for the CBR video quality enhancement task.

This paper proposes a video enhancement method for CBR compressed video. Specifically, we first study the compression method and principles of HEVC. Because the high frequency components are given a larger quantization step in the HEVC encoding system, observed compression artifacts are largely due to the loss of high-frequency information. We design a dual-domain image restoration module, and propose a new multi-scale framework namely Constant Bit Rate video quality Enhancement convolutional neural Network (CBREN). To make use of multi-frame information, a feature alignment and fusion module (FAFM) is applied when the video is sent into the network. Fig. 1 shows some results of videos enhanced by CBREN. The main contributions of this paper are as follows:

- We propose a novel CBREN approach for constant bit rate video quality enhancement. In order to effectively reduce the block distortion of different sizes, CBREN is designed as a three-branch structure, processing the corresponding block compression artifacts in three scales.

- We design a dual-domain restoration module (DRM) to suppress the compression artifacts of HEVC compressed video, which models and removes the artifacts in the pixel-domain and the DCT-domain respectively.

- We propose a two-step quantization table estimation strategy comprising of an IDCT translation unit (ITU) to obtain the base quantization table by a learning method, and a Dynamic Alpha Unit (DAU) to slightly adjust the quantization table according to the content of each frame.

\section{RELATED WORK}

The quality enhancement of compressed video is challenging as it involves a variety of tasks, such as artifact removal, texture restoration, use of multi-frame information. In this section, we briefly discuss several related methods.
Image restoration. Inspired by SRCNN [14], Dong et al. [13] first propose a deep learning-based method for reduction of image compression artifacts. Zheng et al. [72] then introduce a scalable deep network for JPEG compression artifact reduction. Zhang et al. [66] apply discriminative learning methods to the image restoration task. Kim et al. [27] introduce recursive supervision and skip-connection to avoid exploding or vanishing gradients, and propose a very deep neural network for image super-resolution. Tai et al. [49] introduce a 52-convolutional-layer deep network with a residual learning method. Several studies focus on multiscale CNNs inspired by high-level computer vision methods [15, 73, 69]. Mao et al. [15] introduce a skip-connection-based autoencoder with multi-scale strategy. Kim et al. [28] propose a pseudo-blind system for removing compression artifacts for compressed video with unknown quality factor. Waqas $e t$ al. [63] introduce a multi-scale network for a variety of image processing tasks, including image denoising, super resolution, and image enhancement. Zheng et al. [69] introduce multiple dilated convolutions formulating receptive pyramids for efficient JPEG deblocking.

Frequency domain learning. The enhancement of compressed images in the Discrete Cosine Transform (DCT) domain has been proven effective. Foi et al. [16] apply pointwise Shape-Adaptive DCT (SADCT) to reduce the blocking and ringing effects caused by JPEG compression. After Zhao et al. [34] first proposed the dual-domain learning method, it was rapidly incorporated into deep learning approaches and achieved good results. Wang et al. [55] use a DNN model instead of a PCA module for feature extraction, and achieve performance improvement. Guo et al. [20] introduce a dualdomain learning architecture and build a 30-convolutionallayer network (DDCN) to utilize both the pixel-domain features and DCT-domain priors. Zhang et al. [65] develop DNCNN, which can be applied to a variety of image restoration tasks. Zheng et al. [71, 70, 2] propose an implicit dualdomain convolution network (IDCN) to reduce JPEG image compression artifacts. IDCN uses a pixel position marker map and a quantization table as input. Different from the traditional dual-domain learning method, which applies a DCT transform into DCT-domain, a DCT-domain loss is directly estimated by the extracted features without requiring a DCT transform. IDCN shows good performance in enhancing quality of JPEG compressed images.

Compressed video enhancement. For the task of compressed video quality enhancement, many enhancement methods based on convolutional neural networks have been proposed to replace the loop filter in HEVC intra coding [11, 29, 24, 38, 62]. Li et al. [32] propose a deep residual network for enhancing quality of the decoded intra frames of HEVC. Chauvet et al. [8] introduce a lightweight adaptive postfilter network. Wang et al. [53] propose a Deep CNNbased Auto Decoder to remove the distortion of compressed video. Yang et al. [60] introduce DS-CNN for video quality enhancement. Huang et al. [24] propose a novel network which filters the luminance channel separately from the pair of chrominance channels. Jin et al. [25] and Wang et al. [52] use a generative adversarial network to remove visual artifacts 
in compressed video. He et al. [22] design a network that uses the partition information produced by the encoder to guide the quality enhancement process. These methods can be regarded as single frame quality enhancement methods, and do not take advantage of the highly similar features between adjacent frames.

Multi-frame processing. The motivation of multi-frame based methods is to utilize the additional information appearing in adjacent frames to improve the quality of the current frame. Temporal alignment plays an important role in multiframe based methods. Several methods compute optical flow to estimate the motion between video frames for alignment [61, 19, 37, 36, 35]. The Multi-Frame Quality Enhancement (MFQE) [61] network and MFQE 2.0 [19] improve the quality by utilizing nearest Peak Quality Frames (PQFs) that have higher quality. TOFlow [58] reveals that the standard optical flow is not the optimal motion representation for video restoration. DUF [26] and TDAN [51] circumvent the problem by implicit motion compensation and surpass the flow-based methods. EDVR [54] uses a pyramid and cascade structure to deal with larger motion, and achieves better feature alignment and fusion. IRDNN [21] proposes an inter-layer restoration deep neural network for scalable high efficiency video coding (SHVC). The patch-wise spatial-temporal quality enhancement network (PSTQE) [12] employs a temporal and spatial-wise attention-based feature distillation structure to adaptively exploit adjacent patches for distilling patch-wise temporal features. The spatial-temporal fusion convolutional neural network (STEF-CNN) [57] employs spatial and temporal information to improve the performance of the in-loop filter in HEVC.

\section{Challenges for HEVC CBR encoding}

\section{A. Quantization method of HEVC}

The quantization in CBR encoding transforms the image in the spatial domain into the DCT-domain, expresses it in the form of coefficients, and encodes these transformation coefficients [6]. It can reduce the correlation between the coefficients and effectively eliminate the spatial redundancy of the image. Quantization of the DCT coefficients achieves compression but also typically results in information loss [18].

In order to partition moving objects more accurately and reduce the amount of transform computation, HEVC uses a more flexible coding scheme than JPEG [42, 31]. The coding unit $(\mathrm{CU})$ is the basic pixel block for the coding operation [1]. The size of the CU can be $4 \times 4,8 \times 8,16 \times 16$, and $32 \times 32$ according to the video content [5, 43], as shown in Fig. 2(a). And the CU partition is applied recursively [44], as shown in Fig. 2(b). In order to reduce the storage space of quantization parameters, only $4 \times 4$ and $8 \times 8$ quantization matrices are given in HEVC, and $16 \times 16$ and $32 \times 32$ quantization matrices are obtained by scaling these two matrices [5].

Because the CUs have many different sizes, and the distribution is very irregular, it is difficult to directly segment the image into the basic coding pixel blocks for further processing.

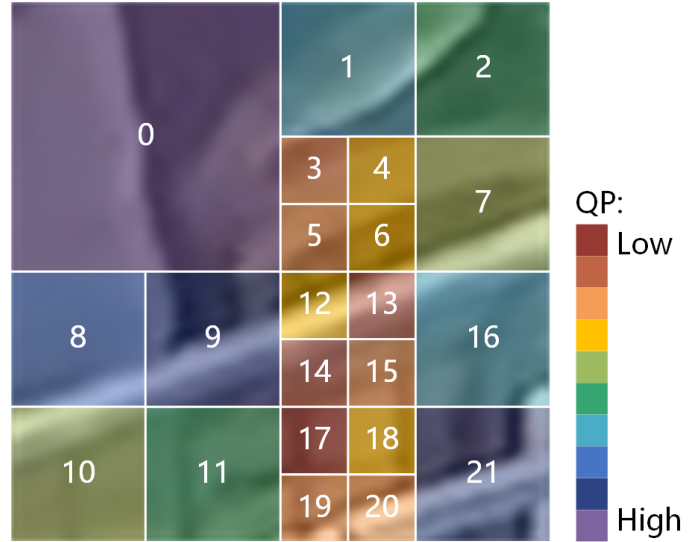

(a)

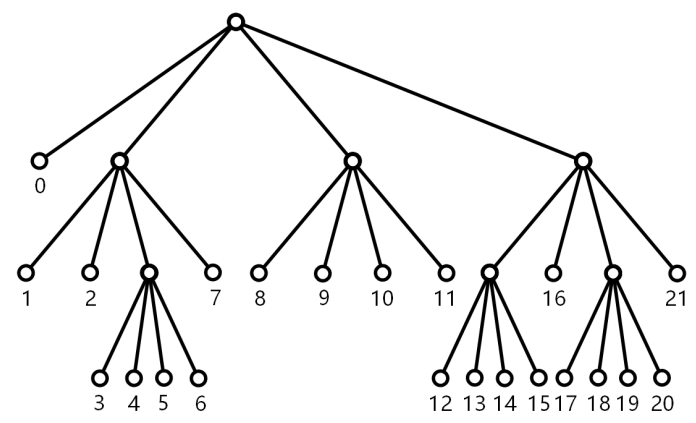

(b)

Fig. 2: Schematic diagram of CU partition, QP value distribution and its corresponding quadtree representation. (a) Schematic diagram of $\mathrm{CU}$ partition and $\mathrm{QP}$ value distribution. (b) The representation of the corresponding quadtree.

\section{B. Bit rate control strategy of $C B R$}

In the CBR compression mode, each frame is encoded at a constant bit rate. When facing large motions, the codeword needs to be reduced by increasing the quantization parameters, which leads to image quality degradation. As shown in Fig. 2(a), the compressed video (in CBR mode) has varying quantization parameters in different locations of the same frame. Modeling, and recovering of, the quantization loss cannot achieve satisfactory results using a fixed quantization parameter matrix.

\section{PROPOSED METHOD}

\section{A. Image Degradation Model}

In order to solve the issues mentioned above, we propose a new method to model quantization degradation for HEVC CBR compressed video. It's a common strategy to use a convolution layer to first transform the degraded image into the feature domain, where artifacts are then estimated and removed. The HEVC compression artifact is main results from the quantization operations applied to the DCT coefficients. Assuming $\Theta$ is a $p \times p$ sized DCT coefficient matrix, the quantization of $\Theta$ can be expressed as:

$$
\Theta^{*}=\Theta-\xi
$$


where $\xi$ denotes the quantization loss. So that the artifacts in the pixel domain $N_{p}$ and feature domain $N_{f}$ can be respectively expressed as:

$$
\begin{gathered}
N_{p}=T_{D C T}^{-1}(\xi) \\
N_{f}=\mathcal{C}\left(N_{p}\right)=\mathcal{C}\left(T_{D C T}^{-1}(\xi)\right)
\end{gathered}
$$

where $T_{D C T}^{-1}$ denotes the IDCT and $\mathcal{C}$ denotes a convolution operation. Noticing that both $T_{D C T}^{-1}$ and $\mathcal{C}$ are linear operations, $N_{f}$ is linearly correlated to $\xi$. Therefore, we can obtain $N_{f}$ by first estimating the $\xi$, and then sequentially applying $T_{D C T}^{-1}$ and $\mathcal{C}$. Because $\xi$ is produced by the quantization of HEVC, we can further express it as:

$$
\xi=\theta \cdot T_{Q p}
$$

where $T_{Q p}$ is a $p \times p$ sized quantization table under the specified quantization parameter (Qp), and $\theta$ is the relative quantization loss, which is a $p \times p$ sized matrix resulting from rounding in the quantization process, and satisfies:

$$
-0.5<\theta_{i}<0.5, \quad \forall \theta_{i} \in \theta
$$

Therefore, the estimation of $\xi$ can be converted to the estimation of $\theta$ and $T_{Q p}$.

Estimating $\theta$. Similar to previous studies directly using convolution layers to estimate the artifacts in the feature domain, we use convolution layers to estimate $\theta$. To match the prior described in Eq. 5, we introduce the following constraints:

$$
\mathcal{L}\left(\theta_{i}\right)=\left\{\begin{array}{cc}
-0.5 & \theta_{i}<-0.5 \\
\theta_{i} & -0.5 \leq \theta_{i} \leq 0.5 \\
0.5 & \theta_{i}>0.5
\end{array}\right.
$$

Although there is no supervision to explicitly ensure the accuracy of the estimated DCT coefficients loss, back-propagation during training will guide the network achieve a roughly good estimation [71].

Estimating $T_{Q p}$. HEVC obtains the $T_{Q p}$ under the specified $Q P$ as:

$$
T_{Q p}=\alpha(Q p) \cdot T_{f}+\beta(Q p)
$$

where $T_{f}$ is the basic quantization table defined by HEVC, $\alpha(Q p)$ and $\beta(Q p)$ are the linear coefficient and bias respectively to adjust the quantization table along with the $Q p$. Though $Q p$ is visible in the decoding stage, it is generally unknown in most post-processing applications. Moreover, as described in Sec. III-B, dynamically adjusting $Q p$ is a common way for bit rate control. So we need to dynamically estimate $\alpha(Q p)$ and $\beta(Q p)$ in the restoration process.

In most CBR modes, the value of $Q p$ is pre-assigned to a limited range, so we only need to estimate $\alpha(Q p)$ and $\beta(Q p)$ in that range. We propose a two-step strategy for efficiently estimating $T_{Q p}$, which can be described as:

$$
T_{\text {est }}=\alpha_{\text {est }} \cdot T_{\text {base }}
$$

where $T_{e s t}$ denotes the final estimated $T_{Q p}, \alpha_{e s t}$ is a dynamic factor adjusted using the input frames and $T_{\text {base }}$ is a basic quantization table learned in the training stage. Since the value

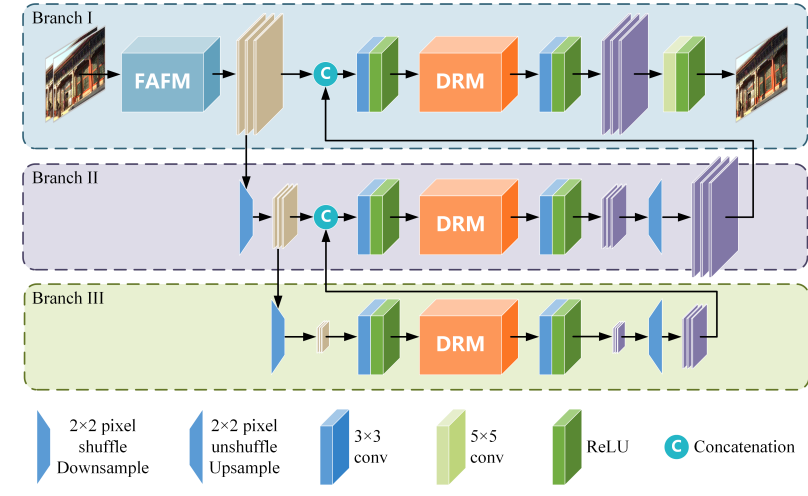

Fig. 3: Architecture of our proposed constant bit rate video quality enhancement convolutional neural network (CBREN).

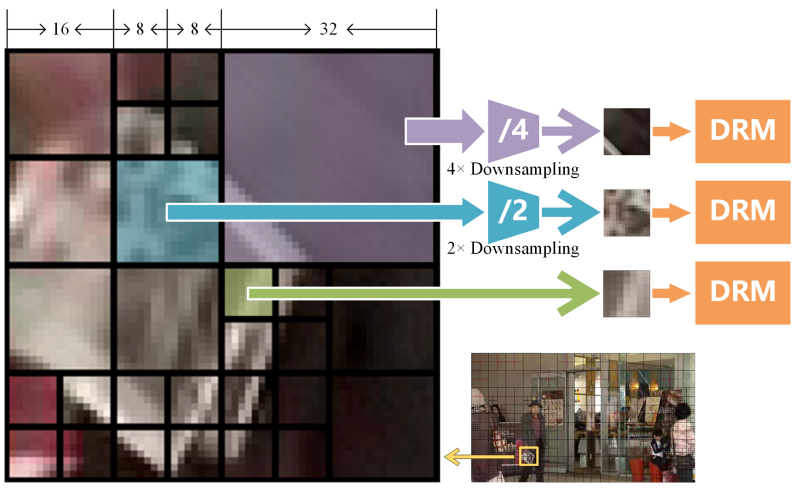

Fig. 4: Schematic diagram of multi-scale processing strategy.

of $Q p$ is pre-assigned to a limited range, we first obtain a basic quantization table $\left(T_{\text {base }}\right)$ to represent the general quantization steps with learnable parameters described below. Then $\alpha_{e s t}$ is introduced to slightly adjust the quantization steps based on the input frames. Following Eq. 7, we use a learning-based model for $T_{\text {base }}$, which can be expressed as:

$$
T_{\text {base }}=\alpha_{\text {base }} \cdot T_{f}+\beta_{\text {base }}
$$

where $\alpha_{\text {base }}$ and $\beta_{\text {base }}$ are both learnable matrices with the same shape of $T_{f}$, with $\alpha_{\text {base }}$ initialized to 1 and $\beta_{\text {base }}$ initialized to 0 . This completes the modeling of the HEVC quantitative loss.

\section{B. Overview of Our Approach}

Block artifact removal using a single scale network cannot achieve satisfactory results, as the basic pixel blocks quantized in the HEVC compression process have many sizes, and the distribution of blocks is irregular. To address this challenge, we design a multi-scale network, named CBREN, illustrated in Fig. 3. CBREN's architecture has three branches, and each branch has a dual-domain restoration module (DRM) to process the block artifacts of the corresponding size. CBREN takes five adjacent compressed frames as input and generates a high-quality output frame. Through the feature alignment and fusion module (FAFM) in Branch I, each neighboring frame is aligned to the reference frame, and the features from all frames are merged. The merged features are used as input to the following modules. 


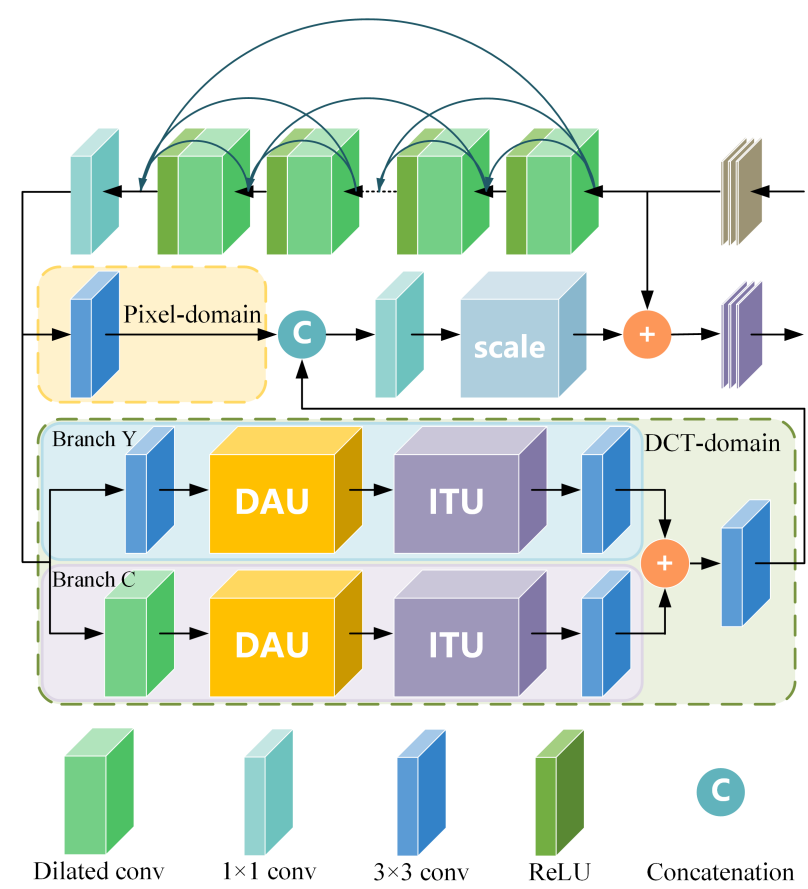

Fig. 5: Architecture of our proposed dual-domain restoration module (DRM).

The basic pixel block size for transform and quantization has four sizes: $4 \times 4,8 \times 8,16 \times 16$, and $32 \times 32$. Among them the $4 \times 4$ pixel block size is very small, and the degradation resulting from compression distortion is not obvious, so we skip the $4 \times 4$ pixel block and set the basic processing scale of DRM as $8 \times 8$. Then we adopt the multi-scale architecture shown in Fig. 4 to approach the $16 \times 16$ and $32 \times 32$ processing via $\times 2$ and $\times 4$ downsampling. Since $32 \times 32$ is the largest pixel block, there is no block artifact with larger size, so it is unnecessary to process it on a larger scale. We have also proved this in the subsequent experiments.

\section{Feature Alignment and Fusion module}

In the video compressed by the CBR mode, the frames with large motion usually have block artifacts and information degradation. The missing information may exist in adjacent frames, which can help enhance the current frame. We adopt the alignment and fusion methods proposed in EDVR [54] to construct a feature alignment and fusion module (FAFM) for multiple frames. First, features are aligned from the low scale to the high scale with pyramid cascaded deformable convolution. Next, a temporal attention mechanism is introduced to produce different weights to different features by calculating the element-by-element relationship between the features of the reference frame and adjacent frames, and then the features are merged according to the weights.

\section{Dual-domain Restoration Module}

The architecture of the dual-domain restoration module (DRM) is shown in Fig. 5. First, feature extraction is performed by a set of receptive field pyramids composed of

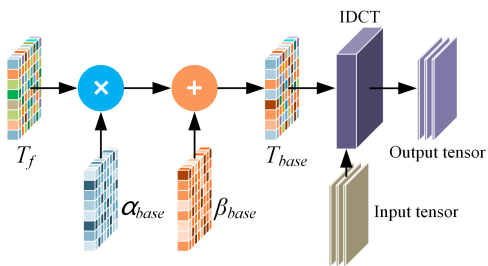

Fig. 6: Structure of our proposed IDCT translation unit (ITU).

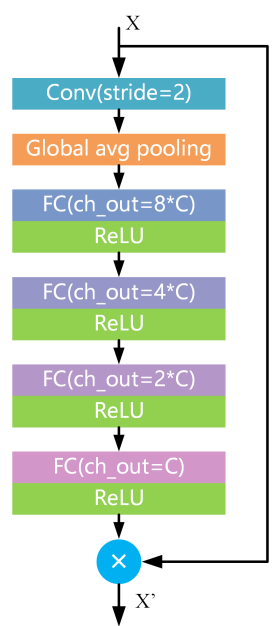

(a)

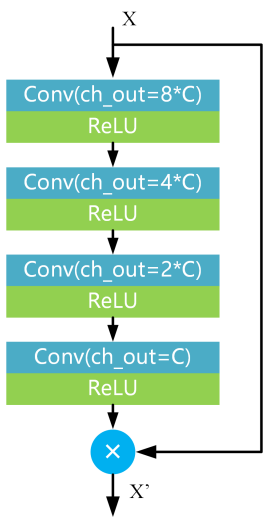

(b)

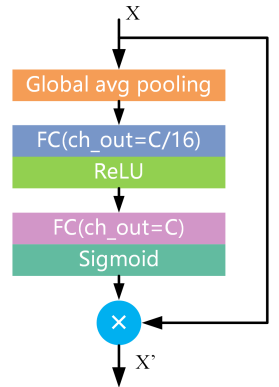

(c)
Fig. 7: Comparison of DAU, DAU-CU and SE Block. Here $C$ denotes the number of channels of the input feature. (a) DAU. (b) DAU-CU. (c) SE Block.

densely connected $3 \times 3$ dilated convolution layers. The densely connected structure is used to extract high-dimensional features. The dilated convolution is introduced to enlarge the receptive field for the following process. A $1 \times 1$ convolution layer is used as the feature fusion layer to fuse the convolution features generated by dense connection. Next, the pixeldomain restoration branch and the DCT-domain restoration branch are introduced in parallel to obtain the artifact representations from pixel domain and DCT-domain respectively. Finally, the outputs of the two branches are concatenated and fused by a $1 \times 1$ convolution layer to obtain the final artifact representation. Specifically, a scaling layer whose factor is set to 0.1 is applied to the final artifact representation before adding to the input of the DRM for avoiding gradient explosion [33].

For the two branches, we used a $3 \times 3$ convolution layer to make a direct estimation for the $N_{f}$ from the fused features in the pixel-domain branch, which will be further fused with the output of the DCT-domain branch. In the DCT-domain, we follow Eq. 3 to make the estimation. YUV420 is the default pre-processing mode for general video compression. Therefore we respectively make the estimation from all three channels. Noticing that the two chrominance channels share the same basic quantization table (denoted as $T_{f}^{C}$ in Fig. 5) and sampling rate, only a single estimation of the two chrominance channels is necessary. We denote the sub-branch for estimating the quantization loss of luminance channel as Branch $Y$, 
and the sub-branch for estimating quantization loss of two chrominance channels as Branch $C$. In both sub-branches, a $3 \times 3$ convolution layer is firstly introduced to estimate the relative quantization loss $\xi$, and the constraint defined in Eq. 6 is then applied. Because the sampling rate of two chrominance channels is half of the luminance channel, we introduce a $3 \times 3$ dilated convolution layer with an expansion ratio of 2 to match this difference. After that, the Dynamic Alpha Unit (DAU) adaptively obtains the $\alpha_{\text {est }}$ based on the input and the IDCT translation unit (ITU) makes the final transform defined in Eq. 3. These are sequentially applied to obtain the corresponding artifact representations in the feature domain.

IDCT Transform Unit. The structure of the proposed ITU is shown in Fig. 6. We adopt the convolution-based IDCT to construct the ITU. Specifically, we reshape the $(8 \times 8) \times(8 \times$ 8) sized IDCT kernel to a $1 \times 1 \times 64 \times 64$ sized tensor, so that we can using a $1 \times 1$ convolution to simulate the IDCT operation. Suggested by [71], all $\alpha_{\text {est }}, \alpha_{\text {base }}$ and $\beta_{\text {est }}$ could be directly applied on the IDCT kernel to avoid the unnecessary point-wise tensor multiplication. However, because $\alpha_{e s t}$ is a dynamic variable, we only directly apply the $\alpha_{\text {base }}$ and $\beta_{\text {est }}$ on the IDCT kernel while the $\alpha_{\text {est }}$ is multiplied on the estimated $\theta$ for the easy multi-batch training.

Following Eq. 9, we introduce two learnable matrices with the shape of $8 \times 8$ serving as $\alpha_{\text {base }}$ and $\beta_{\text {base. }}$. The basic quantization tables of two sub-branches $T_{f}^{Y}$ and $T_{f}^{C}$ for branch $\mathrm{Y}$ and branch $\mathrm{C}$ are pre-assigned as they are defined in the encoding application and will not be updated in the training stage. After the IDCT, a $3 \times 3$ convolution layer is stacked to obtain the artifact representation in the feature domain.

Dynamic Alpha Unit. Based on the multiscale architecture, we can make a point-wise estimation to approach the CUwise $\alpha_{e s t}$ estimation. However this operation is inefficient. From Eq. 4, both $\theta$ and $T_{Q p}$ requires a point-wise estimation. Since there is no additional supervision to separately guide the two estimations, this dual pixel-wise estimation will make no difference to the single pixel-wise estimation. We will demonstrate this in the following experimental section. Because the estimation for $\theta$ must be point-wise, we adopt a frame-wise estimation for $T_{Q p}$. As we previously introduced, $\alpha_{e s t}$ should directly multiply on the $\theta$, we propose a channel attention-like structure to make such an operation.

Fig. 7(a) illustrates the structure of the proposed DAU. We use a $3 \times 3$ convolution layer with stride of $2 \times 2$ to enhance the input feature map and then use global average pooling to obtain the global features for the following framewise $\alpha_{\text {est }}$ estimation. Then multiple fully connected layers are sequentially stacked to estimate $\alpha_{e s t}$. Because the $T_{Q p}$ is not sparse, we adopt channel increment rather than channel reduction [23] to achieve an over complete estimation.

\section{EXPERIMENTS}

\section{A. Datasets}

In previous research on video processing, development and evaluation were usually carried out on private data sets, and there is a lack of a standard public data set. We use a new
TABLE I: The specific parameters of the two groups of bit rates settings.

\begin{tabular}{ccccc}
\hline \hline Sequence & Resolution & Frame rate(fps) & Low(kbps) & High(kbps) \\
\hline Reference & $960 \times 536$ & 30 & 200 & 800 \\
\hline A & $2560 \times 1600$ & 30 & 1592 & 6368 \\
\hline \multirow{2}{*}{ B } & \multirow{2}{*}{$1920 \times 1080$} & 24 & 645 & 2580 \\
& & 50 & 1343 & 5372 \\
& \multirow{2}{*}{$* 32 \times 480$} & 60 & 1612 & 6448 \\
\hline & & 50 & 155 & 620 \\
& \multirow{2}{*}{$416 \times 240$} & 60 & 259 & 1036 \\
D & 30 & 50 & 39 & 1240 \\
& & 60 & 65 & 156 \\
E & $1280 \times 720$ & 60 & 78 & 312 \\
\hline \hline
\end{tabular}

video data set proposed by NTIRE2021 ${ }^{1}$, which contains a large number of videos collected from YouTube. The data set consists of 200 training videos, 20 validation videos and 20 test videos. Each video has more than 100 consecutive frames. Since the ground truth of the validation video and the test video were not available in the competition, we selected 10 representative videos and selected 20 consecutive frames from each video as our validation data set. The remaining 190 training videos were used as our training data set. We use the HEVC standard test sequence as test data set.

Because x.265 is get much closer to industrial applications than the official reference software HM, we apply the x.265 HEVC encoder to compress all HEVC standard test sequences in both CBR mode and CQP mode. Considering most of CQPoriented methods are evaluated with HM, we also compress the test sequences with HM16.0 in CQP mode for a fair comparison. The quantization parameters of all CQP compressed videos is set to 37 . In CBR mode, if we set all the videos to the same bit rate, the compression degree of videos with different frame rates and resolutions are different, and the quality of the compressed video varies.

In order to ensure that all videos have an appropriate degree of compression, we set a fixed compression ratio with reference to the training data set, and set different bit rates for the videos according to the frame rate and resolution. Then we compare the quality of the compressed video with different parameters in CQP and CBR modes, as shown in Figure 7. We can see that the video compressed by CQP mode always has a very stable quality, and the compressed video quality in CBR mode is closely related to the content of the video. Even with the same bitrate per pixel, there is still a large quality difference between different videos. Considering that in practical applications, CBR mode is usually used in scenarios with limited bandwidth, the video compressed in CBR mode usually has poor quality, so we decided to use two sets of lower bit rates as the test set. The specific bit rate settings of each video in the test sequence are shown in TABLE I.

\footnotetext{
${ }^{1}$ https://data.vision.ee.ethz.ch/cvl/ntire21/
} 

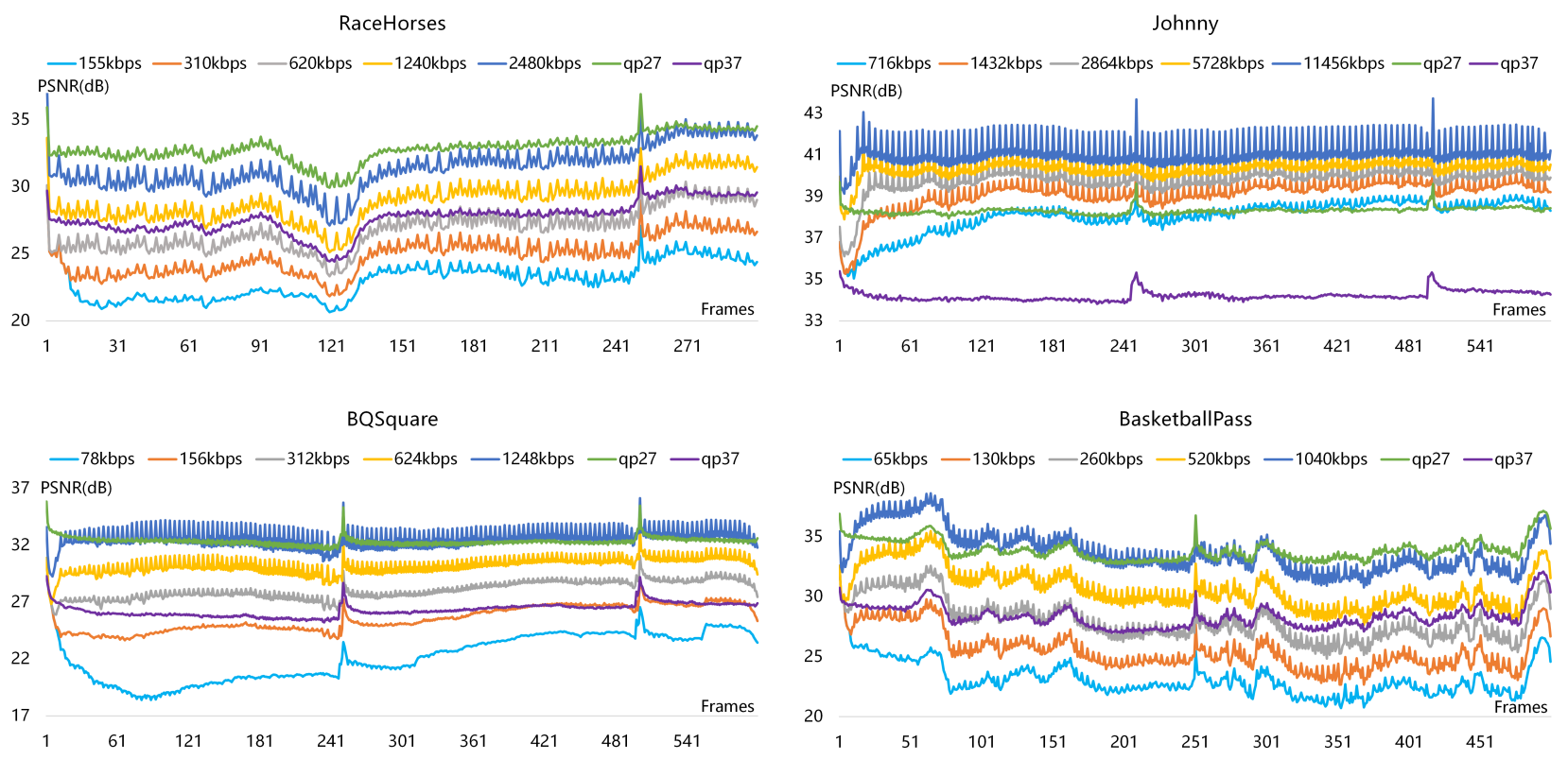

Fig. 8: PSNR curves of compressed video by various compression modes with various parameters.

TABLE II: Quantitative comparison for handling CBR task. Best results (red), second best (blue).

\begin{tabular}{|c|c|c|c|c|c|c|c|c|c|c|c|c|c|c|c|c|c|}
\hline & \multirow{3}{*}{ Video } & \multicolumn{8}{|c|}{ Low bit rate } & \multicolumn{8}{|c|}{ High bit rate } \\
\hline & & \multicolumn{2}{|c|}{$\begin{array}{c}\text { IDCN } \\
\text { TCSVT } 2019\end{array}$} & \multicolumn{2}{|c|}{$\begin{array}{c}\text { EDVR } \\
\text { CVPRW } 2019\end{array}$} & \multicolumn{2}{|c|}{$\begin{array}{c}\text { MIRNet } \\
\text { ECCV } 2020\end{array}$} & \multicolumn{2}{|c|}{$\begin{array}{c}\text { Ours } \\
\text { Proposed }\end{array}$} & \multicolumn{2}{|c|}{$\begin{array}{c}\text { IDCN } \\
\text { TCSVT } 2019\end{array}$} & \multicolumn{2}{|c|}{$\begin{array}{c}\text { EDVR } \\
\text { CVPRW } 2019\end{array}$} & \multicolumn{2}{|c|}{$\begin{array}{c}\text { MIRNet } \\
\text { ECCV } 2020\end{array}$} & \multicolumn{2}{|c|}{$\begin{array}{c}\text { Ours } \\
\text { Proposed }\end{array}$} \\
\hline & & $\triangle \mathrm{PSNR}$ & $\Delta$ SSIM & $\triangle \mathrm{PSNR}$ & $\Delta$ SSIM & $\triangle \mathrm{PSNR}$ & $\Delta$ SSIM & $\triangle \mathrm{PSNR}$ & $\Delta$ SSIM & $\triangle \mathrm{PSNR}$ & $\Delta$ SSIM & $\triangle \mathrm{PSNR}$ & $\Delta$ SSIM & $\triangle \mathrm{PSNR}$ & $\Delta$ SSIM & $\triangle \mathrm{PSNR}$ & $\Delta$ SSIM \\
\hline \multirow{2}{*}{ A } & Traffic & 0.414 & 0.0126 & 0.133 & 0.0082 & 0.390 & 0.0125 & 0.286 & 0.0127 & 0.325 & 0.0071 & 0.049 & 0.0042 & 0.319 & 0.0072 & 0.430 & 0.0084 \\
\hline & PeopleOnStreet & 0.213 & 0.0209 & 0.814 & 0.0540 & 0.298 & 0.0262 & 1.017 & 0.0629 & 0.704 & 0.0201 & 0.507 & 0.0166 & 0.719 & 0.0201 & 1.030 & 0.0262 \\
\hline \multirow{5}{*}{ B } & Kimono & 0.436 & 0.0178 & 0.327 & 0.0147 & 0.427 & 0.0181 & 0.564 & 0.0227 & 0.436 & 0.0110 & 0.307 & 0.0090 & 0.451 & 0.0111 & 0.496 & 0.0122 \\
\hline & ParkScene & 0.338 & 0.0144 & 0.400 & 0.0145 & 0.312 & 0.0137 & 0.492 & 0.0197 & 0.398 & 0.0104 & 0.238 & 0.0077 & 0.368 & 0.0098 & 0.486 & 0.0123 \\
\hline & Cactus & 0.448 & 0.0155 & 0.448 & 0.0144 & 0.464 & 0.0153 & 0.628 & 0.0203 & 0.342 & 0.0081 & 0.193 & 0.0041 & 0.374 & 0.0076 & 0.455 & 0.0097 \\
\hline & BQTerrace & 0.417 & 0.0091 & 0.104 & 0.0040 & 0.417 & 0.0091 & 0.203 & 0.0067 & 0.233 & 0.0046 & 0.115 & 0.0031 & 0.315 & 0.0059 & 0.351 & 0.0062 \\
\hline & BasketballDrive & 0.476 & 0.0183 & 0.638 & 0.0235 & 0.592 & 0.0221 & 0.822 & 0.0275 & 0.521 & 0.0113 & 0.290 & 0.0075 & 0.597 & 0.0121 & 0.659 & 0.0126 \\
\hline \multirow{4}{*}{$\mathrm{C}$} & RaceHorses & 0.273 & 0.0235 & 0.529 & 0.0368 & 0.316 & 0.0261 & 0.633 & 0.0411 & 0.604 & 0.0243 & 0.486 & 0.0154 & 0.585 & 0.0232 & 0.724 & 0.0276 \\
\hline & BQMall & 0.488 & 0.0224 & 0.650 & 0.0279 & 0.539 & 0.0251 & 0.854 & 0.0349 & 0.770 & 0.0150 & 0.717 & 0.0178 & 0.723 & 0.0146 & 0.934 & 0.0174 \\
\hline & PartyScene & 0.176 & 0.0141 & 0.291 & 0.0210 & 0.237 & 0.0190 & 0.410 & 0.0294 & 0.549 & 0.0190 & 0.579 & 0.0175 & 0.564 & 0.0197 & 0.714 & 0.0239 \\
\hline & BasketballDrill & 0.526 & 0.0276 & 0.696 & 0.0342 & 0.676 & 0.0347 & 1.004 & 0.0439 & 0.827 & 0.0185 & 0.643 & 0.0173 & 0.854 & 0.0193 & 1.035 & 0.0224 \\
\hline \multirow{4}{*}{ D } & RaceHorses & 0.190 & 0.0208 & 0.525 & 0.0418 & 0.225 & 0.0236 & 0.615 & 0.0476 & 0.632 & 0.0272 & 0.604 & 0.0181 & 0.614 & 0.0265 & 0.796 & 0.0337 \\
\hline & BQSquare & 0.288 & 0.0183 & 0.325 & 0.0210 & 0.327 & 0.0204 & 0.450 & 0.0263 & 0.801 & 0.0135 & 0.923 & 0.0195 & 0.817 & 0.0143 & 1.019 & 0.0171 \\
\hline & BlowingBubbles & 0.208 & 0.0173 & 0.349 & 0.0220 & 0.248 & 0.0196 & 0.460 & 0.0281 & 0.524 & 0.0187 & 0.487 & 0.0191 & 0.500 & 0.0185 & 0.675 & 0.0241 \\
\hline & BasketballPass & 0.229 & 0.0187 & 0.523 & 0.0353 & 0.308 & 0.0257 & 0.710 & 0.0435 & 0.723 & 0.0233 & 0.690 & 0.0170 & 0.734 & 0.0234 & 1.010 & 0.0309 \\
\hline \multirow{4}{*}{ E } & FourPeople & 0.626 & 0.0084 & 0.270 & 0.0070 & 0.648 & 0.0084 & 0.722 & 0.0099 & 0.073 & 0.0044 & 0.381 & 0.0218 & 0.286 & 0.0050 & 0.445 & 0.0055 \\
\hline & Johnny & 0.353 & 0.0046 & -0.668 & 0.0017 & 0.454 & 0.0044 & 0.280 & 0.0049 & -0.075 & 0.0027 & 0.272 & 0.0065 & 0.137 & 0.0021 & 0.282 & 0.0037 \\
\hline & KristenAndSara & 0.313 & 0.0061 & -0.404 & 0.0040 & 0.466 & 0.0059 & 0.435 & 0.0070 & -0.362 & 0.0035 & 0.361 & 0.0296 & 0.175 & 0.0040 & 0.231 & 0.0047 \\
\hline & Average & 0.356 & 0.0161 & 0.331 & 0.0214 & 0.408 & 0.0183 & 0.588 & 0.0272 & 0.446 & 0.0135 & 0.436 & 0.0140 & 0.508 & 0.0136 & 0.654 & 0.0166 \\
\hline
\end{tabular}

\section{B. Training details}

The network has a complex structure and a large number of parameters. The effect of the DRM in the network depends heavily on the performance of the FAFM. The whole end-toend training may fail to converge and it is hard to achieve good results. Therefore, we first train the FAFM alone and include it as a pre-trained model, and then the complete model is trained. The pre-training and full training use the same training parameters.

In the network, 64 channels are used in all trainable convolution layers without any specifications. Each branch has a DRM with the same structure. In each DRM, there are six dilated convolutions in the densely connected structure, and the dilation rates of these convolutions are $\{1,2,3,3,2,1\}$ respectively. This settings ensure that the dense connection structure provides enough receptive field for the following $8 \times 8$ IDCT-based processing.

We use five consecutive video frame patches as the input of the training. The patch is randomly cropped to $96 \times 96$ pixels from the video frame, then data augmentation is carried out through the horizontal and vertical flipping and the random $90^{\circ}$ rotation. L2 loss is used as the loss function, the batch size is set to 8 , Adam is used as the training optimizer, and the initial learning rate is set to $10^{-4}$. When the objective evaluation index of the validation of five consecutive epochs 


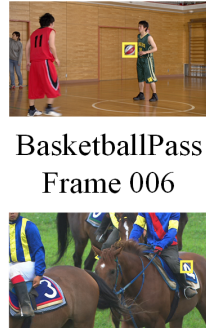

RaceHorses Frame 249

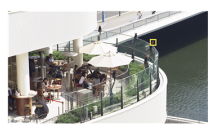

ParkScene Frame 145
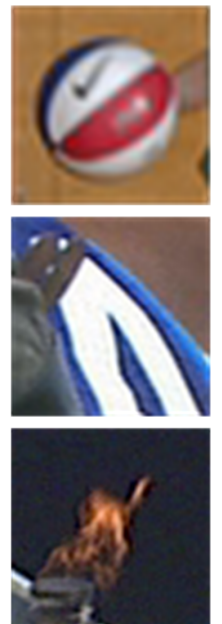

Raw
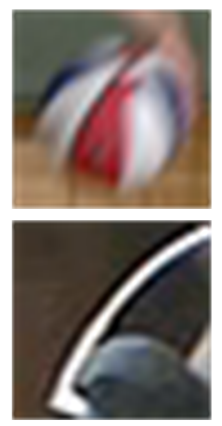

RaceHorse

Frame 140

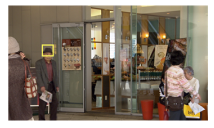

BQMall

Frame 231

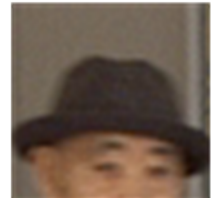

Raw
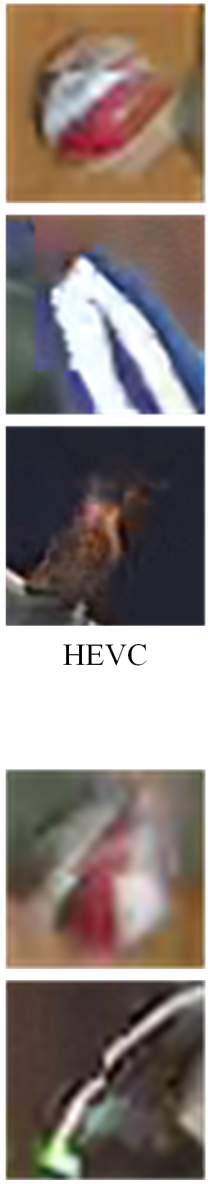

HEVC

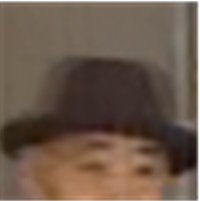

HEVC
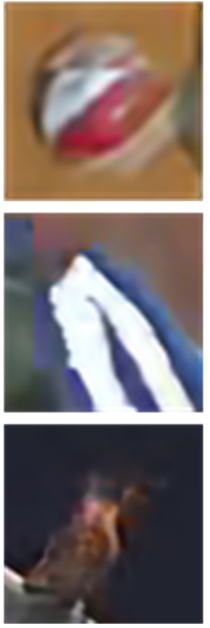

ARCNN
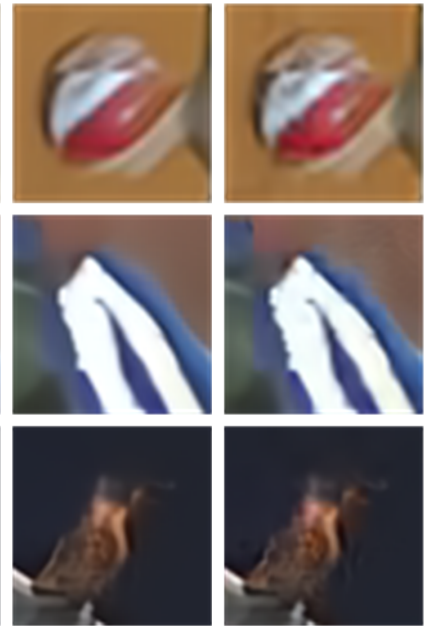

MIRNet

(a)
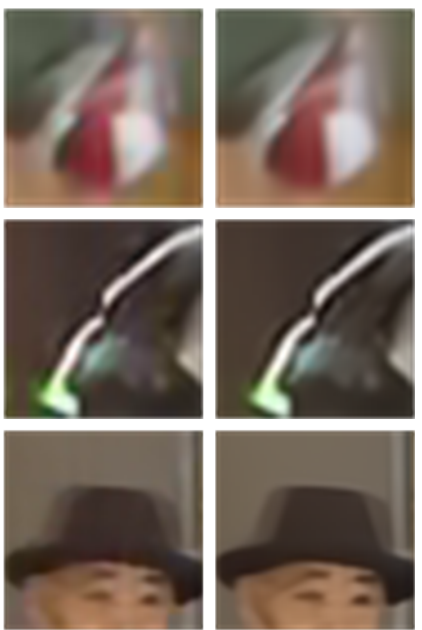

ARCNN
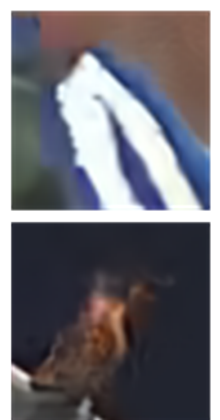

IDCN
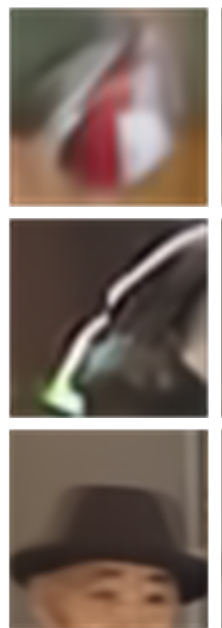

IDCN

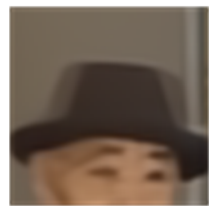

MIRNet
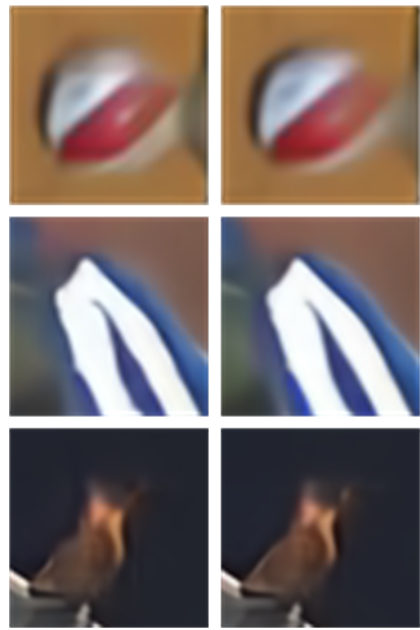

EDVR

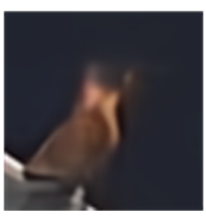

Ours
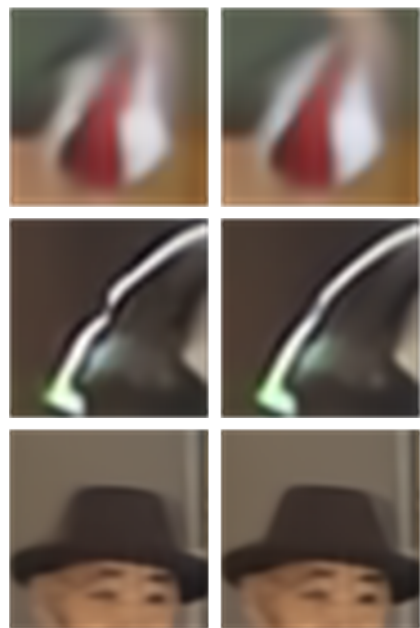

EDVR
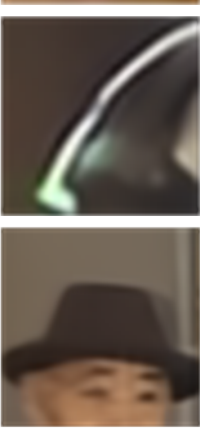

Ours

(b)

Fig. 9: Subjective evaluation with the CBR compression. (a) Compressed in Low bit rate. (b) Compressed in High bit rate.

does not further improve, the learning rate is reduced to half of the current value, and the training is stopped when the learning rate is lower than $10^{-6}$.

We use the Pytorch framework for our implementation, and train on an Nvidia RTX 3090 GPU.

\section{Performance of our approach}

In this section, we test our method in CBR and CQP mode and compare it with other methods. We use the $\triangle$ PSNR and $\triangle$ SSIM as the objective evaluation measure to capture the video enhancement, which measures the PSNR and SSIM gap between the enhanced and original compressed sequences.

Quality enhancement on CBR compressed video. The existing video quality enhancement schemes generally focus on CQP compressed video. However, compared with CQP compressed video, the enhancement CBR compressed video is more challenging.
We compared CBREN with the state-of-the-art CNN based image/video enhancement methods on CBR compressed video quality enhancement. Considering that there is no special video enhancement scheme for CBR compressed video, we reproduced several representative image/video enhancement methods including ARCNN [53], IDCN [71], EDVR [54], and MIRNet [63] for evaluation. For fairness, all these methods are reproduced with the same training settings provided in original publications and trained on the same datasets as our method.

TABLE II gives the average RGB $\triangle$ PSNR and $\triangle$ SSIM on all frames of each HEVC test sequence. The subjective comparison between ARCNN, MIRNet, IDCN, EDVR and CBREN is shown in Fig. 9. The results show that our method has a very significant effect in improving the quality of CBR compressed video, and it is superior to the existing methods to a large extent.

Quality enhancement of CQP compressed video. In order to further evaluate the performance of CBREN, we also com- 


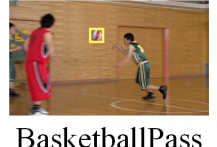

Frame 60

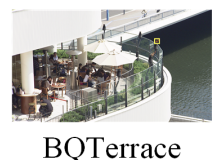

Frame 60

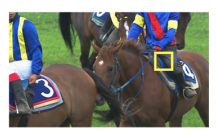

RaceHorses

Frame 250
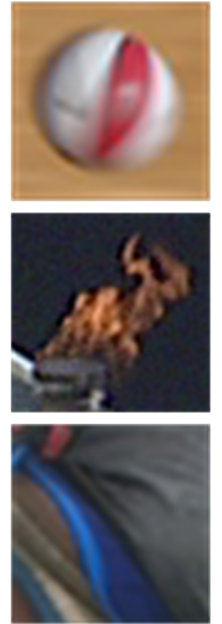

Raw
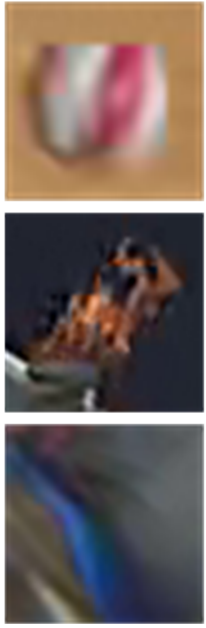

HEVC
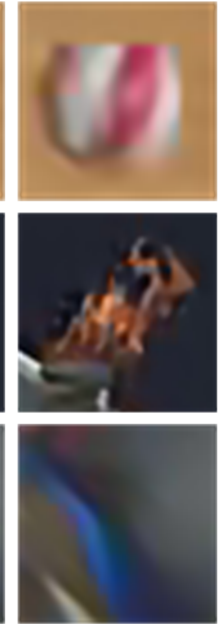

ARCNN
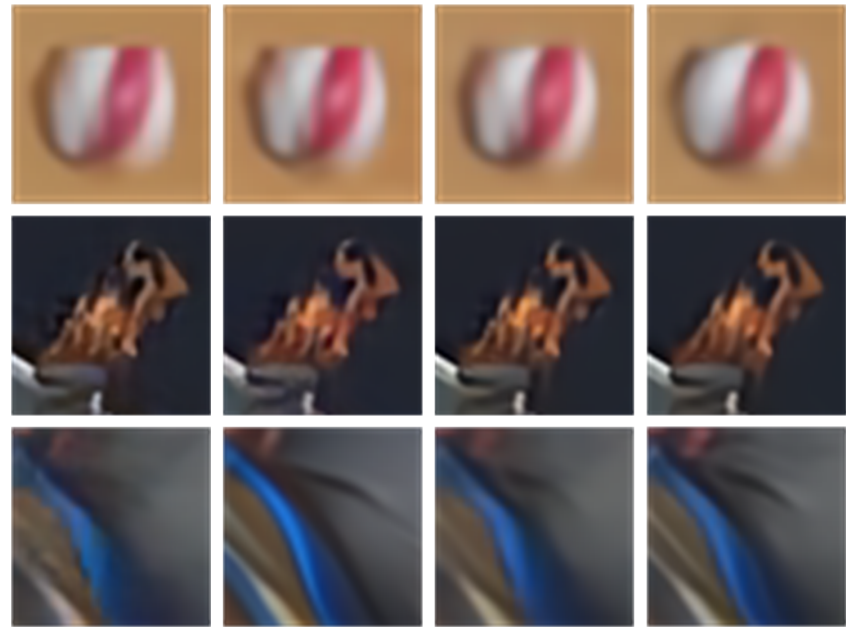

MIRNet

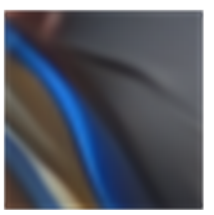

IDCN

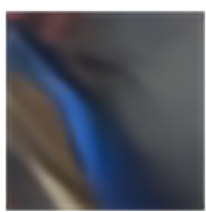

EDVR

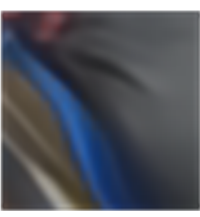

Ours

Fig. 10: Subjective evaluation with $\mathrm{qp}=37 \mathrm{CQP}$ compression.

TABLE III: Quantitative comparison for the CQP task. Best results (red), second best (blue).

\begin{tabular}{|c|c|c|c|c|c|c|c|c|c|c|c|}
\hline \multirow{2}{*}{ Encoder } & \multirow{2}{*}{ Group } & \multicolumn{2}{|c|}{$\begin{array}{c}\text { IDCN } \\
\text { TCSVT } 2019\end{array}$} & \multicolumn{2}{|c|}{$\begin{array}{c}\text { EDVR } \\
\text { CVPR } 2019\end{array}$} & \multicolumn{2}{|c|}{$\begin{array}{c}\text { MFQE } 2.0 \\
\text { TPAMI } 2019\end{array}$} & \multicolumn{2}{|c|}{$\begin{array}{c}\text { MIRNet } \\
\text { ECCV } 2020\end{array}$} & \multicolumn{2}{|c|}{$\begin{array}{c}\text { Ours } \\
\text { Proposed }\end{array}$} \\
\hline & & $\triangle \mathrm{PSNR}$ & $\Delta$ SSIM & $\triangle \mathrm{PSNR}$ & $\Delta$ SSIM & $\triangle \mathrm{PSNR}$ & $\Delta$ SSIM & $\triangle \mathrm{PSNR}$ & $\Delta$ SSIM & $\triangle \mathrm{PSNR}$ & $\Delta$ SSIM \\
\hline \multirow{5}{*}{ x.265 } & A & 0.442 & 0.0136 & 0.239 & 0.0128 & 0.399 & 0.0090 & 0.373 & 0.0131 & 0.631 & 0.0137 \\
\hline & B & 0.297 & 0.0094 & 0.252 & 0.0083 & 0.232 & 0.0066 & 0.376 & 0.0098 & 0.479 & 0.0117 \\
\hline & $\mathrm{C}$ & 0.390 & 0.0153 & 0.328 & 0.0141 & 0.242 & 0.0075 & 0.469 & 0.0169 & 0.644 & 0.0170 \\
\hline & $\mathrm{D}$ & 0.383 & 0.0152 & 0.434 & 0.0156 & 0.240 & 0.0083 & 0.441 & 0.0175 & 0.703 & 0.0221 \\
\hline & $\mathrm{E}$ & 0.418 & 0.0090 & 0.440 & 0.0080 & 0.424 & 0.0053 & 0.386 & 0.0090 & 0.795 & 0.0103 \\
\hline \multicolumn{2}{|c|}{ Average } & 0.386 & 0.0125 & 0.339 & 0.0118 & 0.307 & 0.0073 & 0.409 & 0.0133 & 0.650 & 0.0150 \\
\hline \multirow{5}{*}{ HM 16.0} & A & 0.413 & 0.0077 & 0.228 & 0.0084 & 0.753 & 0.0130 & 0.366 & 0.0083 & 0.591 & 0.0079 \\
\hline & B & 0.231 & 0.0045 & 0.150 & 0.0054 & 0.475 & 0.0098 & 0.260 & 0.0062 & 0.434 & 0.0074 \\
\hline & $\mathrm{C}$ & 0.297 & 0.0074 & 0.334 & 0.0092 & 0.489 & 0.0110 & 0.370 & 0.0107 & 0.628 & 0.0098 \\
\hline & $\mathrm{D}$ & 0.333 & 0.0084 & 0.465 & 0.0114 & 0.548 & 0.0133 & 0.396 & 0.0109 & 0.726 & 0.0109 \\
\hline & $\mathrm{E}$ & 0.381 & 0.0053 & 0.719 & 0.0092 & 0.697 & 0.0083 & 0.470 & 0.0081 & 0.985 & 0.0097 \\
\hline \multicolumn{2}{|c|}{ Average } & 0.331 & 0.0067 & 0.379 & 0.0087 & 0.592 & 0.0111 & 0.372 & 0.0088 & 0.673 & 0.0091 \\
\hline
\end{tabular}

pare to the state-of-the-art methods on CQP compressed video of QP = 37, including MFQE2.0 [19], IDCN [71], EDVR [54] and MIRNet [63]. We tested the compared methods for both x.265 and HM16.0. All the the compared methods are trained on the x.265 based training dats and directly tested on the HM16.0 compressed videos. However MFQE2.0 could not be trained on our device due to the code compatibility issues, so we adopt the publicly released model trained for HM16.0 to make comparisons.

Table III shows the Y channel $\triangle \mathrm{PSNR}$ and $\triangle$ SSIM results on all frames of each group of the HEVC test sequences. Fig. 10 shows results side-by-side for subjective visual assessment. Benefiting from the multi-frame strategy and the multi-scale architecture with DRMs, our CBREN method is superior to other methods in the x.265 based test. For the HM16.0 based test, our CBREN approach still achieves the best PSNR score and the second best SSIM score, that only the specifically trained MFEQ2.0 achieves better SSIM score than our CBREN. This result demonstrates that CBREN is also competent for $\mathrm{CQP}$ video quality enhancement and shows good compatibility for both x.265 and HM16.0 encoders.

\section{Ablation study}

In this part, we study the specific effect and role of each part of our method. The following experiments all use unified model parameters and experimental settings. We continue to train on the CBR data set provided by NTIRE2021. In order to improve the speed of the experiment, we directly train the complete model, and other training details are the same as those mentioned above. We use group D in the HEVC standard test sequence for CBR compression as the test set, and take the average RGB $\triangle$ PSNR and $\triangle$ SSIM of all four videos to evaluate performance.

Multi-frame strategy. In this part, we verify the necessity and effectiveness of using aligned and fused multi-frame information to improve the video quality enhancement. Therefore, we use three adjacent frames, five adjacent frames, seven adjacent frames and a single frame without the FAFM to enhance the video. The experimental results are shown in the Table IV. We can see that our method only uses a single frame 
TABLE IV: Performance comparison of Multi-frame and Multi-scale options.

\begin{tabular}{|c|c|c|c|c|c|c|c|c|c|}
\hline \multicolumn{4}{|c|}{ Multi-frame } & \multicolumn{4}{|c|}{ Multi-scale } & \multirow{2}{*}{$\triangle \mathrm{PSNR}$} & \multirow{2}{*}{$\Delta \mathrm{SSIM}$} \\
\hline 1 & 3 & 5 & 7 & $\times 1$ & $\times 2$ & $\times 4$ & $\times 8$ & & \\
\hline$\checkmark$ & \multirow{9}{*}{$\checkmark$} & & \multirow{9}{*}{$\checkmark$} & $\checkmark$ & $\checkmark$ & $\checkmark$ & & 0.445 & 0.0163 \\
\hline & & & & $\checkmark$ & $\checkmark$ & $\checkmark$ & & 0.508 & 0.0339 \\
\hline & & $\checkmark$ & & $\checkmark$ & $\checkmark$ & $\checkmark$ & & 0.521 & 0.0346 \\
\hline & & & & $\checkmark$ & $\checkmark$ & $\checkmark$ & & 0.359 & 0.1170 \\
\hline & & $\checkmark$ & & $\checkmark$ & & & & 0.508 & 0.0343 \\
\hline & & $\checkmark$ & & & $\checkmark$ & & & 0.493 & 0.0333 \\
\hline & & $\checkmark$ & & & & $\checkmark$ & & 0.477 & 0.0267 \\
\hline & & $\checkmark$ & & & $\checkmark$ & $\checkmark$ & & 0.502 & 0.0341 \\
\hline & & $\checkmark$ & & $\checkmark$ & $\checkmark$ & $\checkmark$ & $\checkmark$ & 0.527 & 0.0346 \\
\hline
\end{tabular}

TABLE V: Performance comparison of DRM, ITU and DAU options.

\begin{tabular}{|c|c|c|c|c|c|c|c|}
\hline \multicolumn{2}{|c|}{ DRM } & \multirow{2}{*}{ ITU } & \multicolumn{3}{|c|}{ DAU } & \multirow{2}{*}{$\triangle \mathrm{PSNR}$} & \multirow{2}{*}{$\Delta$ SSIM } \\
\hline DCT Y & DCT C & & DAU & DAU-CU & SE Block & & \\
\hline & & $\checkmark$ & $\checkmark$ & & & 0.472 & 0.0323 \\
\hline$\checkmark$ & & $\checkmark$ & $\checkmark$ & & & 0.520 & 0.0344 \\
\hline$\checkmark$ & $\checkmark$ & & $\checkmark$ & & & 0.450 & 0.0315 \\
\hline$\checkmark$ & $\checkmark$ & $\checkmark$ & $\checkmark$ & & & 0.521 & 0.0346 \\
\hline$\checkmark$ & $\checkmark$ & $\checkmark$ & & $\checkmark$ & & 0.521 & 0.0342 \\
\hline$\checkmark$ & $\checkmark$ & $\checkmark$ & & & $\checkmark$ & 0.504 & 0.0337 \\
\hline$\checkmark$ & $\checkmark$ & $\checkmark$ & & & & 0.513 & 0.0342 \\
\hline
\end{tabular}

as the input, and the $\triangle$ PSNR of the test video is only $0.445 \mathrm{~dB}$. Compared with the method of feature alignment and fusion of three consecutive frames, the $\triangle \mathrm{PSNR}$ of the video can reach $0.508 \mathrm{~dB}$. When five consecutive frames are used as input, the enhancement effect is further improved.

When seven consecutive frames are input into FAFM, the enhancement effect is weaker than that of a single frame. The quality correlation of adjacent frames in HEVC compressed video is closely related to the intra prediction mechanism. The GOP (group of picture) length of HEVC is usually less than five frames. Different GOPs have different macroblock partition methods, and the same information becomes different features after compression. Therefore, alignment and fusion of many consecutive frames will introduce useless features and degrade the enhancement effect.

The above research proves that it is necessary to use aligned and fused multi-frame information in our method. Using FAFM to obtain multi-frame information can significantly improve the enhancement effect. However, for optimal results, there is a limit to the number of frames that should be used.

Multi-scale architecture strategy. We verify the effectiveness of the multi-scale structure in the main structure of the model. The experimental results are shown in the Table IV. For comparison, we build the models using 1, 2 and 4 single scale scaling features respectively. We observe that using different scales will result in a different enhancement effect. When the scaling scale is 1 , the effect of the network is the best.

Next, we build a multi-scale model, and test the model using $\times 2 \times 4, \times 1 \times 2 \times 4$ and $\times 1 \times 2 \times 4 \times 8$ multi-scale schemes. From the experimental results, we know that a multi-scale structure can achieve a better result than a single scale structure. The model of $\times 1 \times 2 \times 4$ has an excellent effect, while $\times 1 \times 2 \times 4 \times 8$ adds a branch to it, but there is almost no improvement on the performance. This verifies our theory that the three scale branches of $\times 1 \times 2 \times 4$ just enhance the pixel blocks with sizes of $8 \times 8,16 \times 16$ and $32 \times 32$ respectively.

Dual-domain restoration module. In order to analyze the effect of the DRM, we first developed three networks which are learned (1) only in the pixel-domain, (2) the pixeldomain and the DCT-domain with only the luminance channel, and (3) both the pixel-domain and the DCT-domain with luminance and chrominance channels. Then we investigate the effects of the ITU and DAU. Specifically, to fully evaluate the performance of the DAU, we develop another two networks by replacing DAU with an SE block [23] and a CU-wise $\alpha_{\text {est }}$ estimation (DAU-CU). For DAU-CU, we remove the first convolution with $2 \times 2$ stride and global average pooling layers and replace the rest of the fully connected layers by the corresponding $3 \times 3$ convolution layers. Fig. 7(b) and Fig. 7(c) illustrate the structures of the SE block and DAU-CU.

The experimental results are shown in Tab. V. Utilizing full information from both the pixel-domain and the DCTdomain leads to better performance. Without the DCT-based processing provided by ITU, the channel attention-like DAU by itself cannot utilize the priors of the DCT coefficients quantization, and leads to a $0.071 \mathrm{~dB}$ performance reduction. Because the quantization table of the HEVC is totally nonsparse, the sparsity hypothesis-based SE block fails to make an accurate estimation for $\alpha_{\text {est }}$. Also as claimed in Sec. IV-D, introducing two pixel-wise estimations at the same time is quite inefficient, as using the CU-wise estimation doesn't make any improvement but consumes much more computational resource. Generally, the proposed components in DRM are proven to be effective and efficient.

\section{CONCLUSION}

In this paper, we propose a constant bit rate video quality enhancement convolutional neural network (CBREN) for video quality enhancement. Our method can also be applied to the CQP video enhancement task, and is superior to the existing methods. The DRM is proposed to learn the prior of compression loss and eliminate it in the pixel-domain and DCTdomain. The DRM has two steps in the process of quantitative loss estimation: rough linear correction and feature-based realtime prediction. The ITU is used to correct the quantization table of CBR compressed video to an appropriate range. On this basis, the DAU predicts the quantization table in real time according to the content of each frame, and makes local finetuning on this basis. Finally, a large number of experimental results show that our CBREN method significantly improves the quality of CBR compressed video, and it is also better than the existing methods for CQP video enhancement.

\section{ACKNOWLEDGEMENT}

This work is supported by The National Key Research and Development Program of China SY2020YFB1406604, 
National Nature Science Foundation of China (62001146, 61931008, 61671196, 61701149, 61801157, 61971268, 61901145, 61901150, 61972123), National Natural Science Major Foundation of Research Instrumentation of PR China under Grants 61427808, Zhejiang Province Nature Science Foundation of China (LR17F030006, Q19F010030), 111 Project, No. D17019.

\section{REFERENCES}

[1] S. Ahn, B. Lee, and M. Kim. "A Novel Fast CU Encoding Scheme Based on Spatiotemporal Encoding Parameters for HEVC Inter Coding". In: IEEE Transactions on Circuits and Systems for Video Technology 25.3 (2015), pp. 422-435.

[2] Zheng Bolun et al. "Learning Frequency Domain Priors for Image Demoireing". In: IEEE Transactions on Pattern Analysis and Machine Intelligence (2021).

[3] F. Bossen et al. "HEVC Complexity and Implementation Analysis". In: IEEE Transactions on Circuits and Systems for Video Technology 22.12 (2013), pp. 16851696.

[4] Frank Bossen et al. "HEVC complexity and implementation analysis". In: IEEE Transactions on Circuits and Systems for Video Technology 22.12 (2012), pp. 16851696.

[5] Madhukar Budagavi, Arild Fuldseth, and Gisle Bjøntegaard. "HEVC transform and quantization". In: High Efficiency Video Coding (HEVC). Springer, 2014, pp. 141-169.

[6] Po-Chun Chang et al. "A DCT/DST-based error propagation-free data hiding algorithm for HEVC intracoded frames". In: Journal of Visual Communication and Image Representation 25.2 (2014), pp. 239-253.

[7] Huibin Chang, Michael K Ng, and Tieyong Zeng. "Reducing artifacts in JPEG decompression via a learned dictionary". In: IEEE transactions on signal processing 62.3 (2013), pp. 718-728.

[8] Antoine CHAUVET et al. "Fast image quality enhancement for HEVC by postfiltering via shallow neural networks". In: IIEEJ Transactions on Image Electronics and Visual Computing 7.1 (2019), pp. 2-12.

[9] Yunjin Chen, Wei Yu, and Thomas Pock. "On learning optimized reaction diffusion processes for effective image restoration". In: Proceedings of the IEEE conference on computer vision and pattern recognition. 2015, pp. 5261-5269.

[10] Kostadin Dabov et al. "Image denoising by sparse 3-D transform-domain collaborative filtering". In: IEEE Transactions on image processing 16.8 (2007), pp. 2080-2095.

[11] Yuanying Dai, Dong Liu, and Feng Wu. "A convolutional neural network approach for post-processing in HEVC intra coding". In: International Conference on Multimedia Modeling. Springer. 2017, pp. 28-39.

[12] Qing Ding et al. "Patch-Wise Spatial-Temporal Quality Enhancement for HEVC Compressed Video". In: IEEE Transactions on Image Processing 30 (2021), pp. 64596472.
[13] Chao Dong et al. "Compression artifacts reduction by a deep convolutional network". In: Proceedings of the IEEE International Conference on Computer Vision. 2015, pp. 576-584.

[14] Chao Dong et al. "Learning a deep convolutional network for image super-resolution". In: European conference on computer vision. Springer. 2014, pp. 184-199.

[15] Lian-Feng Dong et al. "Learning deep representations using convolutional auto-encoders with symmetric skip connections". In: 2018 IEEE International Conference on Acoustics, Speech and Signal Processing (ICASSP). IEEE. 2018, pp. 3006-3010.

[16] Alessandro Foi, Vladimir Katkovnik, and Karen Egiazarian. "Pointwise shape-adaptive DCT as an overcomplete denoising tool". In: Proc. 2005 Int. TICSP Workshop Spectral Meth. Multirate Signal Process., SMMSP 2005. 2005, pp. 164-170.

[17] Alessandro Foi, Vladimir Katkovnik, and Karen Egiazarian. "Pointwise shape-adaptive DCT for highquality denoising and deblocking of grayscale and color images". In: IEEE transactions on image processing 16.5 (2007), pp. 1395-1411.

[18] Wei Gao et al. "DCT coefficient distribution modeling and quality dependency analysis based frame-level bit allocation for HEVC'. In: IEEE Transactions on Circuits and Systems for Video Technology 26.1 (2015), pp. 139-153.

[19] Zhenyu Guan et al. "MFQE 2.0: A new approach for multi-frame quality enhancement on compressed video". In: IEEE transactions on pattern analysis and machine intelligence (2019).

[20] Jun Guo and Hongyang Chao. "Building dual-domain representations for compression artifacts reduction". In: European Conference on Computer Vision. Springer. 2016, pp. 628-644.

[21] Gang He et al. "Interlayer Restoration Deep Neural Network for Scalable High Efficiency Video Coding”. In: IEEE Transactions on Circuits and Systems for Video Technology (2021).

[22] Xiaoyi He et al. "Enhancing HEVC compressed videos with a partition-masked convolutional neural network". In: 2018 25th IEEE International Conference on Image Processing (ICIP). IEEE. 2018, pp. 216-220.

[23] Jie $\mathrm{Hu}, \mathrm{Li}$ Shen, and Gang Sun. "Squeeze-andexcitation networks". In: Proceedings of the IEEE conference on computer vision and pattern recognition. 2018, pp. 7132-7141.

[24] Hongyue Huang, Ionut Schiopu, and Adrian Munteanu. "Frame-wise CNN-based filtering for intra-frame quality enhancement of HEVC videos". In: IEEE Transactions on Circuits and Systems for Video Technology (2020).

[25] Zhipeng Jin et al. "Quality enhancement for intra frame coding via cnns: An adversarial approach". In: 2018 IEEE International Conference on Acoustics, Speech and Signal Processing (ICASSP). IEEE. 2018, pp. 1368-1372. 
[26] Younghyun Jo et al. "Deep video super-resolution network using dynamic upsampling filters without explicit motion compensation". In: Proceedings of the IEEE conference on computer vision and pattern recognition. 2018, pp. 3224-3232.

[27] Jiwon Kim, Jung Kwon Lee, and Kyoung Mu Lee. "Deeply-recursive convolutional network for image super-resolution". In: Proceedings of the IEEE conference on computer vision and pattern recognition. 2016, pp. 1637-1645.

[28] Yoonsik Kim et al. "A pseudo-blind convolutional neural network for the reduction of compression artifacts". In: IEEE Transactions on Circuits and Systems for Video Technology 30.4 (2019), pp. 1121-1135.

[29] Shiba Kuanar, Christopher Conly, and KR Rao. "Deep learning based HEVC in-loop filtering for decoder quality enhancement”. In: 2018 Picture Coding Symposium (PCS). IEEE. 2018, pp. 164-168.

[30] Jani Lainema et al. "Intra coding of the HEVC standard". In: IEEE transactions on circuits and systems for video technology 22.12 (2012), pp. 1792-1801.

[31] B. Lee, M. Kim, and T. Q. Nguyen. "A Frame-Level Rate Control Scheme Based on Texture and Nontexture Rate Models for High Efficiency Video Coding”. In: IEEE Transactions on Circuits and Systems for Video Technology 24.3 (2014), pp. 465-479.

[32] Fan Li, Weimin Tan, and Bo Yan. "Deep residual network for enhancing quality of the decoded intra frames of hevc". In: 2018 25th IEEE International Conference on Image Processing (ICIP). IEEE. 2018, pp. 3918-3922.

[33] Bee Lim et al. "Enhanced deep residual networks for single image super-resolution". In: Proceedings of the IEEE conference on computer vision and pattern recognition workshops. 2017, pp. 136-144.

[34] Xianming Liu et al. "Data-driven sparsity-based restoration of JPEG-compressed images in dual transformpixel domain". In: Proceedings of the IEEE Conference on Computer Vision and Pattern Recognition. 2015, pp. 5171-5178.

[35] Xiandong Meng et al. “A Robust Quality Enhancement Method Based on Joint Spatial-Temporal Priors for Video Coding". In: IEEE Transactions on Circuits and Systems for Video Technology (2020).

[36] Xiandong Meng et al. "BSTN: An Effective Framework for Compressed Video Quality Enhancement". In: 2020 IEEE Conference on Multimedia Information Processing and Retrieval (MIPR). IEEE. 2020, pp. 320-325.

[37] Xiandong Meng et al. "Flow-guided temporal-spatial network for HEVC compressed video quality enhancement". In: 2020 Data Compression Conference (DCC). IEEE. 2020, pp. 384-384.

[38] Zhaoqing Pan et al. "Efficient in-loop filtering based on enhanced deep convolutional neural networks for HEVC". In: IEEE Transactions on Image Processing 29 (2020), pp. 5352-5366.

[39] Robert Pepper. Cisco visual networking index (VNI) global mobile data traffic forecast update. Tech. rep.
Cisco, Tech. Rep., Feb. 2013. Accessed: Jul. 10, 2019.[Online]. Available ..., 2013.

[40] Rosewarne et al. "Overview of the Range Extensions for the HEVC Standard: Tools, Profiles, and Performance". In: IEEE Transactions on Circuits and Systems for Video Technology (2016).

[41] D. Schroeder et al. "Efficient multi-rate video encoding for HEVC-based adaptive HTTP streaming". In: IEEE Transactions on Circuits and Systems for Video Technology 28.1 (2016), pp. 1-1.

[42] Liquan Shen, Zhaoyang Zhang, and Ping An. "Fast CU size decision and mode decision algorithm for HEVC intra coding". In: IEEE Transactions on Consumer Electronics 59.1 (2013), pp. 207-213.

[43] Liquan Shen et al. "Fast TU size decision algorithm for HEVC encoders using Bayesian theorem detection". In: Signal Processing: Image Communication 32 (2015), pp. 121-128.

[44] Yunyu Shi, Zhiyong Gao, and Xiaoyun Zhang. "Early TU split termination in HEVC based on quasi-zeroblock". In: 3rd International Conference on Electric and Electronics. Atlantis Press. 2013, pp. 450-454.

[45] Gary J Sullivan et al. "Overview of the high efficiency video coding (HEVC) standard". In: IEEE Transactions on circuits and systems for video technology 22.12 (2012), pp. 1649-1668.

[46] Gary J Sullivan et al. "Standardized extensions of high efficiency video coding (HEVC)". In: IEEE Journal of selected topics in Signal Processing 7.6 (2013), pp. 1001-1016.

[47] Pavel Svoboda et al. "Compression artifacts removal using convolutional neural networks". In: arXiv preprint arXiv:1605.00366 (2016).

[48] Vivienne Sze, Madhukar Budagavi, and Gary J Sullivan. "High efficiency video coding (HEVC)". In: Integrated circuit and systems, algorithms and architectures. Vol. 39. Springer, 2014, p. 40.

[49] Ying Tai, Jian Yang, and Xiaoming Liu. "Image superresolution via deep recursive residual network". In: Proceedings of the IEEE conference on computer vision and pattern recognition. 2017, pp. 3147-3155.

[50] T. K. Tan et al. "Video Quality Evaluation Methodology and Verification Testing of HEVC Compression Performance". In: IEEE Transactions on Circuits and Systems for Video Technology 26.1 (2015), pp. 1-1.

[51] Yapeng Tian et al. "Tdan: Temporally-deformable alignment network for video super-resolution". In: Proceedings of the IEEE/CVF Conference on Computer Vision and Pattern Recognition. 2020, pp. 3360-3369.

[52] Ting Wang et al. "Visual Perception Enhancement for HEVC Compressed Video Using a Generative Adversarial Network". In: 2020 International Conference on UK-China Emerging Technologies (UCET). IEEE. 2020, pp. 1-4.

[53] Tingting Wang, Mingjin Chen, and Hongyang Chao. "A novel deep learning-based method of improving coding efficiency from the decoder-end for HEVC". 
In: 2017 Data Compression Conference (DCC). IEEE. 2017, pp. 410-419.

[54] Xintao Wang et al. "Edvr: Video restoration with enhanced deformable convolutional networks". In: Proceedings of the IEEE/CVF Conference on Computer Vision and Pattern Recognition Workshops. 2019, pp. 00 .

[55] Zhangyang Wang et al. "D3: Deep dual-domain based fast restoration of JPEG-compressed images". In: Proceedings of the IEEE Conference on Computer Vision and Pattern Recognition. 2016, pp. 2764-2772.

[56] Mai Xu et al. "Reducing complexity of HEVC: A deep learning approach". In: IEEE Transactions on Image Processing 27.10 (2018), pp. 5044-5059.

[57] Xiaoyu Xu et al. "Spatial-Temporal Fusion Convolutional Neural Network for Compressed Video Enhancement in HEVC'. In: 2020 Data Compression Conference (DCC). IEEE. 2020, pp. 402-402.

[58] Tianfan Xue et al. "Video enhancement with taskoriented flow". In: International Journal of Computer Vision 127.8 (2019), pp. 1106-1125.

[59] Chenggang Yan et al. "Efficient parallel framework for HEVC motion estimation on many-core processors". In: IEEE Transactions on Circuits and Systems for Video Technology 24.12 (2014), pp. 2077-2089.

[60] Ren Yang, Mai Xu, and Zulin Wang. "Decoder-side HEVC quality enhancement with scalable convolutional neural network". In: 2017 IEEE International Conference on Multimedia and Expo (ICME). IEEE. 2017, pp. 817-822.

[61] Ren Yang et al. "Multi-frame quality enhancement for compressed video". In: Proceedings of the IEEE Conference on Computer Vision and Pattern Recognition. 2018, pp. 6664-6673.

[62] Liangwei Yu et al. "Quality enhancement network via multi-reconstruction recursive residual learning for video coding”. In: IEEE Signal Processing Letters 26.4 (2019), pp. 557-561.

[63] Syed Waqas Zamir et al. "Learning enriched features for real image restoration and enhancement". In: Computer Vision-ECCV 2020: 16th European Conference, Glasgow, UK, August 23-28, 2020, Proceedings, Part XXV 16. Springer. 2020, pp. 492-511.

[64] Hao Zhang and Zhan Ma. "Fast intra mode decision for high efficiency video coding (HEVC)". In: IEEE Transactions on circuits and systems for video technology 4.24 (2014), pp. 660-668.

[65] Kai Zhang et al. "Beyond a gaussian denoiser: Residual learning of deep cnn for image denoising". In: IEEE transactions on image processing 26.7 (2017), pp. 3142-3155.

[66] Kai Zhang et al. "Learning deep CNN denoiser prior for image restoration". In: Proceedings of the IEEE conference on computer vision and pattern recognition. 2017, pp. 3929-3938.

[67] Xiaoshuai Zhang et al. "DMCNN: Dual-domain multiscale convolutional neural network for compression artifacts removal". In: 2018 25th IEEE International
Conference on Image Processing (ICIP). IEEE. 2018, pp. 390-394.

[68] Yongbing Zhang et al. "Residual highway convolutional neural networks for in-loop filtering in HEVC'. In: IEEE Transactions on image processing 27.8 (2018), pp. 3827-3841.

[69] Bolun Zheng et al. "Build receptive pyramid for efficient color image compression artifact reduction". In: Journal of Electronic Imaging 29.3 (2020), p. 033009.

[70] Bolun Zheng et al. "Image Demoireing with Learnable Bandpass Filters". In: Proceedings of the IEEE/CVF Conference on Computer Vision and Pattern Recognition (CVPR). June 2020.

[71] Bolun Zheng et al. "Implicit dual-domain convolutional network for robust color image compression artifact reduction". In: IEEE Transactions on Circuits and Systems for Video Technology 30.11 (2019), pp. 39823994.

[72] Bolun Zheng et al. "S-Net: a scalable convolutional neural network for JPEG compression artifact reduction". In: Journal of Electronic Imaging 27.4 (2018), p. 043037.

[73] Xiaofei Zhou et al. "Edge-Aware Multiscale Feature Integration Network for Salient Object Detection in Optical Remote Sensing Images". In: IEEE Transactions on Geoscience and Remote Sensing (2021).

[74] Xiaofei Zhou et al. "Improving video saliency detection via localized estimation and spatiotemporal refinement". In: IEEE Transactions on Multimedia 20.11 (2018), pp. 2993-3007.

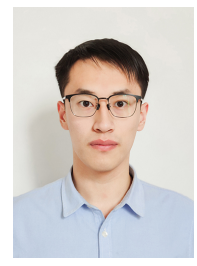

Hengrun Zhao received the B.S. degree from Shenyang Aerospace University in 2016. He is currently pursuing the M.S. degree at the Hangzhou Dianzi University. His research interests mainly include computer vision and image processing.

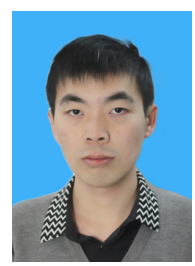

Bolun Zheng received the B.S. and Ph.D. degrees in electronic information technology and instrument from Zhejiang University in 2014 and 2019, respectively. He is currently a lecturer with Hangzhou Dianzi University. His research interests are computer vision, pattern recognition, image processing and embedded parallel computing. 


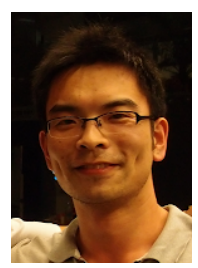

Shanxin Yuan is a Senior Research Scientist in Computer Vision for Huawei Technologies, R\&D.

$\mathrm{He}$ received the $\mathrm{PhD}$ degree from Imperial College London, the MSc degree from the University of Chinese Academy of Sciences, and the BSc degree from China Agricultural University. His research interests are machine learning and computer vision, and he is currently working on low-level vision.

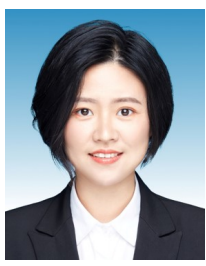

Hua Zhang received the B.S. and Ph.D. degrees in biomedical engineering and instrumentation from Zhejiang University in 2003 and 2009, respectively. She is currently an associate professor at the School of Computer Science, Hangzhou Dianzi University. Her research interests are 3D video encoding/decoding, image quality assessment, and embedded system design.

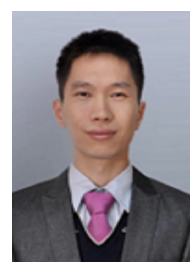

Chenggang Yan received the B.S. degree in computer science from Shandong University in 2008, and the Ph.D. degree in computer science from the Institute of Computing Technology, Chinese Academy of Sciences in 2013. He is currently a Professor with Hangzhou Dianzi University. Before that, he was an Assistant Research Fellow of Tsinghua University. His research interests include intelligent information processing, machine learning, image processing, computational biology, and computational photography.

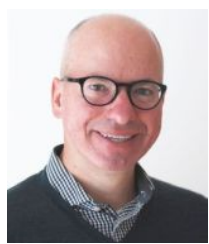

Gregory Slabaugh is Professor of Computer Vision and AI and Director of the Digital Environment Research Institute (DERI) at Queen Mary University of London. Previously, he was Chief Scientist in Computer Vision (EU) for Huawei Technologies R\&D. His research interests include computational photography, medical image computing, and applications of deep learning. 